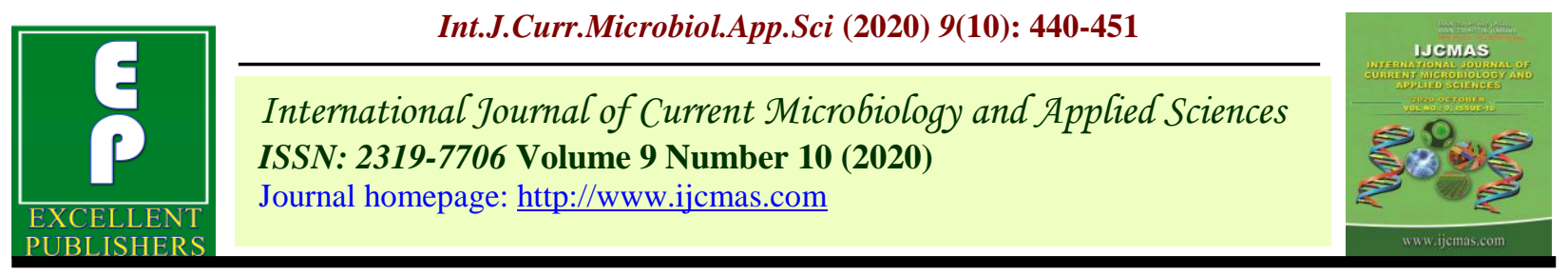

Original Research Article

https://doi.org/10.20546/ijcmas.2020.910.054

\title{
Effect of Sources and Levels of Sulphur on Growth and Yield of kharif Soybean [Glycine max (L.) Merrill]
}

\author{
Janaki Movalia* and S. G. Savalia
}

Department of Agricultural Chemistry and Soil Science, College of Agriculture, Junagadh Agricultural University, Junagadh - 362 001, Gujarat, India

*Corresponding author

Keywords

Soybean, Sulphur sources, Sulphur levels, Growth, Yield

Article Info

Accepted:

07 September 2020

Available Online:

10 October 2020

\section{A B S T R A C T}

\section{Introduction}

Soybean [Glycine max (L.) Merrill] is an introduced and commercially exploited crop in India. Soybean [Glycine max (L.) Merrill] is considered as a miracle crop because of its dual qualities, viz., high protein (40-42\%) and oil content $(20 \%)$ in seed. In India, soybean cultivation was started in 1977. It has high yield potential, wide adaptability, short duration and very high nutritional value having a vast multiplicity of uses as food and industrial products. Being a legume, it fixes a large amount of atmospheric nitrogen in soil. Therefore, soybean crop is known as "Golden Bean", "Miracle Crop", "Wonder Crop" and
"Gold of Soil". Soybean has a very high potential among grain legume crops for combating acute malnutrition. It is a good source of dietary fiber, calcium, magnesium, phosphate, thiamine, riboflavin, niacin, lecithin, potassium, sulphur, vitamins A, B \& $\mathrm{E}$ and essential amino acids like lysine, leucine, methionine and threonine which are required for human body. Soybean protein is mainly rich in amino acids like vegetarians and it is also known as "poor man's meat".

Sulphur as secondary plant nutrient is becoming increasingly important in dryland agriculture as it is the "Master Nutrient" for all oilseed crops and pulses and is rightly 
being called the "Forth Major Plant Nutrient", along with nitrogen, phosphorus and potassium. Sulphur performs many important functions in the plant. It is best known for its role in the synthesis of proteins, oils and vitamins. It is a constituent of three amino acids viz., methionine, cysteine and cystine. Sulphur is also a constituent of S-glycosides (mustard oils), coenzyme A, vitamins, biotine and thiamine as also of iron-sulphur proteins called ferrodoxins. In that sulphur fertilizers are most critical for grain yield, oil and protein synthesis, and improvement of quality of soybean through enzymatic and metabolic efforts (Kumar et al., 1981). It lowers the HCN content of certain crops, promotes nodulation in legumes and produces heavier grains of oilseeds (Tandon, 1987).

\section{Materials and Methods}

A field experiment entitled "Effect of sources and levels of Sulphur on nutrient composition, yield and quality of kharif soybean [Glycine max (L.) Merrill]." was carried out during kharif season of the year 2018 and 2019 at Instructional Farm, Department of Agronomy, College of Agriculture, Junagadh Agricultural University, Junagadh (Fig. 1). The experiment was conducted in C-7 Plot of Instructional Farm, Department of Agronomy, College of Agriculture, Junagadh Agricultural University, Junagadh during Kharif season of 2018 and 2019. The soil was low in available nitrogen (225 and $230 \mathrm{~kg} \mathrm{ha}^{-1}$ in 2018 and 2019, respectively), medium in available phosphorus (30.25 and $33.50 \mathrm{~kg} \mathrm{ha}^{-1}$ in 2018 and 2019, respectively) and high in available potassium (280 and $288 \mathrm{~kg} \mathrm{ha}^{-1}$ in 2018 and 2019 , respectively), low in sulphur (8.94 and $9.80 \mathrm{mg} \mathrm{kg}^{-1}$ in 2018 and 2019, respectively).

Factorial Randomized Block Design with total thirteen treatments replicated thrice was employed in this study. The treatments were assigned to each replication by randomization process. The experiment comprising of total twelve treatment combination in which four sources of sulphur and three sources of sulphur. Absolute control was compared with these treatment combinations in RBD. The four sources viz., $\mathrm{S}_{1}-$ Gypsum $(18-20 \% \mathrm{~S}), \mathrm{S}_{2}$ - Cosavet Fertis ( $80 \%$ S), $S_{3}$ - Elemental sulphur $(100 \% \mathrm{~S}), \mathrm{S}_{4}-$ Bentonite $(90 \% \mathrm{~S})$ and three levels of sulphur viz., $\mathrm{L}_{1}-10 \mathrm{~kg} \mathrm{~S}^{-1}$, $\mathrm{L}_{2}-20 \mathrm{~kg} \mathrm{~S} \mathrm{ha}^{-1}$ and $\mathrm{L}_{3}-30 \mathrm{~kg} \mathrm{~S} \mathrm{ha}^{-1}$ with absolute control. Soybean variety Gujarat Junagadh Soybean -3 was used for sowing with seed rate of $60 \mathrm{~kg} \mathrm{ha}^{-1}$ keeping inter row spacing of $45 \mathrm{~cm}$ on $21^{\text {st }}$ July during 2018 and $25^{\mathrm{h}}$ June 2019. The required quantity of $\mathrm{N} @$ $30 \mathrm{~kg} \mathrm{ha}{ }^{-1}$ and $\mathrm{P} @ 60 \mathrm{~kg} \mathrm{ha}{ }^{-1} \mathrm{P}_{2} \mathrm{O}_{5}$ were applied in the form of urea and DAP, respectively. All other recommended agronomic practices were followed during the period of crop growth. The crop was harvested at maturity on $10^{\text {th }}$ October, 2018 and $5^{\text {th }}$ November, 2019. The data on growth and yield parameters were recorded and the statistical analysis of data of the characters studied by the investigation through the procedure appropriate to the design of the experiment and significance of difference tested by the ' $\mathrm{F}$ ' test (Panse and Sukhatme, 1985).

\section{Results and Discussion}

The results summarized in Table 1 indicated that different sources of sulphur produce significant effect on plant height. The maximum plant height $(45.04,47.37$ and $46.20 \mathrm{~cm}$ ) registered with application of cosavet fertis $\left(\mathrm{S}_{2}\right)$ during year 2018, 2019 and pooled, respectively which was statistically at par (43.28 and $45.61 \mathrm{~cm})$ with elemental sulphur $\left(\mathrm{S}_{3}\right)$ during year 2018 and 2019 Gupta et al., (2003). While, the application of sulphur @ $20 \mathrm{~kg} \mathrm{ha}^{-1}\left(\mathrm{~L}_{2}\right)$ recorded the highest value of plant height (44.29, 46.62 and $45.46 \mathrm{~cm})$ and it was at par $(43.32,45.65$ and $44.48 \mathrm{~cm}$ ) with application of sulphur @ 
$30 \mathrm{~kg} \mathrm{ha}^{-1}\left(\mathrm{~L}_{3}\right)$ during year 2018, 2019 and pooled, respectively. Similar results were also reported by Layek et al., (2014).

Combined application of sulphur sources and levels produced significant effect on plant height (Table 2). The maximum (46.73, 49.06 and $47.90 \mathrm{~cm}$ ) plant height was observed with application of cosavet fertis @ $20 \mathrm{~kg} \mathrm{~S} \mathrm{ha}^{-1}$ $\left(\mathrm{S}_{2} \mathrm{~L}_{2}\right)$ over respective values of control (38.63, 40.80 and $39.71 \mathrm{~cm}$ ) during year 2018, 2019 and pooled, respectively. It was remained at par with application of cosavet fertis@30 kg S ha ${ }^{-1}\left(\mathrm{~S}_{2} \mathrm{~L}_{3}\right)$ and elemental sulphur@20 kg S ha ${ }^{-1}\left(\mathrm{~S}_{3} \mathrm{~L}_{2}\right)$ during year 2018; and cosavet fertis@ $30 \mathrm{~kg} \mathrm{~S} \mathrm{ha}^{-1}\left(\mathrm{~S}_{2} \mathrm{~L}_{3}\right)$ found at par during year 2019 and pooled results.

\section{No. of branches per plant}

The highest no. of branches plant ${ }^{-1}(5.78,5.58$ and 5.68) were recorded with application of cosavet fertis $\left(\mathrm{S}_{2}\right)$ in both years and pooled, respectively. It was remaining at par (5.39 and 5.19) with application of bentonite $\left(\mathrm{S}_{4}\right)$ during both years. These observations are also in agreement with that of Yatheesh et al., (2013).

While, the no. of branches per plant significantly affected by different levels of sulphur (Table 3). The maximum no. of branches per plant (5.58, 5.38 and 5.48) was registered with the application of sulphur @ $20 \mathrm{~kg} \mathrm{ha}^{-1}\left(\mathrm{~L}_{2}\right)$ in both years and pooled, respectively, which was statistically at par (5.33, 5.13 and 5.23) with application of sulphur@30 kg ha ${ }^{-1}\left(\mathrm{~L}_{3}\right)$ during both years and pooled result, respectively Singh et al., (2017).

The application of sulphur @ $20 \mathrm{~kg} \mathrm{ha}^{-1}$ in the form of cosavet fertis $\left(\mathrm{S}_{2} \mathrm{~L}_{2}\right)$ recorded the highest no. of branches per plant (6.10), it was remaining at par with application of sulphur @ $20 \mathrm{~kg} \mathrm{ha}^{-1}$ as a source of gypsum at $\left(\mathrm{S}_{1} \mathrm{~L}_{2}\right)$, sulphur@30 kg ha ${ }^{-1}$ as cosavet fertis $\left(\mathrm{S}_{2} \mathrm{~L}_{3}\right)$ and sulphur@20 kg ha $\mathrm{kg}^{-1}$ as a source of bentonite $\left(\mathrm{S}_{4} \mathrm{~L}_{2}\right)$. The similar result also reported by Singh et al., (2017).

The higher no. of branches per plant (6.20. 6.00 and 6.10) recorded with application of cosavet fertis@20 kg S ha ${ }^{-1}\left(\mathrm{~S}_{2} \mathrm{~L}_{2}\right)$ during year 2018, 2019 and pooled result, respectively over to control value (4.47, 4.40 and 4.43). It was statistically at par with

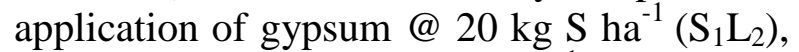
cosavet fertis@30 kg S ha ${ }^{-1}\left(\mathrm{~S}_{2} \mathrm{~L}_{3}\right)$ and bentonite@20 kg S ha ${ }^{-1}\left(\mathrm{~S}_{4} \mathrm{~L}_{2}\right)$ during year 2018 and 2019 and gypsum with $20 \mathrm{~kg} \mathrm{~S} \mathrm{ha}^{-1}$ $\left(\mathrm{S}_{1} \mathrm{~L}_{2}\right)$ and cosavet fertis with $30 \mathrm{~kg} \mathrm{~S}^{-1}$ $\left(\mathrm{S}_{2} \mathrm{~L}_{3}\right)$ in pooled result (Table 4). The similar finding was also noted by Singh et al., (2018).

\section{No. of pods per plant}

The application of cosavet fertis $\left(\mathrm{S}_{2}\right)$ produced the highest pods per plant (41.30, 43.29 and 42.29) during year 2018, 2019 and pooled result, respectively (Table 5). It was statistically at par with application of bentonite $\left(\mathrm{S}_{4}\right)$ with value of $40.73,42.83$ and 41.78 in both years and pooled result, respectively. It was also at par (41.73) with application of gypsum $\left(\mathrm{S}_{1}\right)$ during year 2019 . These results are in close agreement with the findings of Vyas and Khandwe, (2013). The higher pods per plant (41.08, 43.09 and 42.08) were recorded under application of sulphur @ $20 \mathrm{~kg} \mathrm{ha}^{-1}\left(\mathrm{~L}_{2}\right)$ in both years and pooled. It was remaining at par with application of sulphur@30 kg ha ${ }^{-1}\left(\mathrm{~L}_{3}\right)$ with values of $40.35,42.45$ and 41.40 in both years and pooled, respectively. The similar result was reported by Vaiyapuri et al., (2010). 
Table.1 Mean effect of sulphur sources and their levels on plant height $(\mathrm{cm})$ of soybean

\begin{tabular}{|c|c|c|c|}
\hline \multirow[t]{2}{*}{ Treatments } & \multicolumn{3}{|c|}{ Plant height (cm) } \\
\hline & 2018 & 2019 & Pooled \\
\hline \multicolumn{4}{|l|}{ Sulphur sources } \\
\hline$S_{1}-$ Gypsum & 41.22 & 43.96 & 42.59 \\
\hline$S_{2}-$ Cosavet Fertis & 45.04 & 47.37 & 46.20 \\
\hline $\mathbf{S}_{3}-$ Elemental sulphur & 43.28 & 45.61 & 44.45 \\
\hline$S_{4}-$ Bentonite & 42.22 & 44.55 & 43.38 \\
\hline S.Em \pm & 0.73 & 0.63 & 0.48 \\
\hline C.D. at $5 \%$ & 2.14 & 1.85 & 1.38 \\
\hline \multicolumn{4}{|l|}{ Sulphur levels (kg S/ha) } \\
\hline $\mathrm{L}_{1}-\mathbf{1 0}$ & 41.21 & 43.84 & 42.53 \\
\hline $\mathbf{L}_{2}-\mathbf{2 0}$ & 44.29 & 46.62 & 45.46 \\
\hline $\mathbf{L}_{3}-\mathbf{3 0}$ & 43.32 & 45.65 & 44.48 \\
\hline S.Em \pm & 0.63 & 0.55 & 0.42 \\
\hline C.D. at $5 \%$ & 1.86 & 1.60 & 1.19 \\
\hline
\end{tabular}

Table.2 Combined effect of sulphur sources and their levels on plant height $(\mathrm{cm})$ of soybean

\begin{tabular}{|c|c|c|c|}
\hline \multirow[t]{2}{*}{ Treatments } & \multicolumn{3}{|c|}{ Plant height (cm) } \\
\hline & 2018 & 2019 & Pooled \\
\hline $\mathbf{T}_{1}$ : Control & 38.63 & 40.80 & 39.71 \\
\hline$T_{2}: S_{1} L_{1}$ & 41.35 & 43.68 & 42.52 \\
\hline$T_{3}: S_{1} L_{2}$ & 42.57 & 44.90 & 43.74 \\
\hline $\mathbf{T}_{4}: \mathbf{S}_{1} \mathbf{L}_{3}$ & 39.75 & 43.28 & 41.52 \\
\hline$T_{5}: S_{2} L_{1}$ & 43.41 & 45.74 & 44.57 \\
\hline$T_{6}: S_{2} L_{2}$ & 46.73 & 49.06 & 47.90 \\
\hline $\mathbf{T}_{7}: \mathbf{S}_{2} \mathbf{L}_{3}$ & 44.97 & 47.30 & 46.13 \\
\hline$T_{8}: S_{3} L_{1}$ & 43.88 & 46.21 & 45.05 \\
\hline$T_{9}: S_{3} L_{2}$ & 44.33 & 46.66 & 45.50 \\
\hline$T_{10}: S_{3} L_{3}$ & 41.63 & 43.96 & 42.80 \\
\hline$T_{11}: S_{4} L_{1}$ & 43.07 & 45.40 & 44.23 \\
\hline$T_{12}: S_{4} L_{2}$ & 43.52 & 45.85 & 44.69 \\
\hline$T_{13}: S_{4} L_{3}$ & 40.07 & 42.40 & 41.23 \\
\hline \multicolumn{4}{|c|}{ S x L Interaction } \\
\hline S.Em \pm & 1.27 & 1.09 & 0.84 \\
\hline C.D. at $5 \%$ & NS & NS & NS \\
\hline \multicolumn{4}{|c|}{ Control v/s Rest } \\
\hline S.Em \pm & 0.97 & 0.81 & 0.63 \\
\hline C.D. at $5 \%$ & 2.84 & 2.37 & 1.78 \\
\hline C.V. \% & 5.11 & 4.16 & 4.64 \\
\hline \multicolumn{4}{|l|}{$\mathbf{Y} \times \mathbf{T}$} \\
\hline S.Em \pm & \multicolumn{3}{|c|}{1.46} \\
\hline C.D. at 5\% & \multicolumn{3}{|c|}{ NS } \\
\hline
\end{tabular}


Table.3 Mean effect of sulphur sources and their levels on no. of branches per plant of soybean

\begin{tabular}{|c|c|c|c|}
\hline \multirow[t]{2}{*}{ Treatments } & \multicolumn{3}{|c|}{ No. of branches plant ${ }^{-1}$} \\
\hline & 2018 & 2019 & Pooled \\
\hline \multicolumn{4}{|l|}{ Sulphur sources } \\
\hline $\mathbf{S}_{1}-\mathbf{G y p s u m}$ & 5.10 & 4.90 & 5.00 \\
\hline $\mathbf{S}_{2}-$ Cosavet Fertis & 5.78 & 5.58 & 5.68 \\
\hline$S_{3}-$ Elemental sulphur & 4.93 & 4.73 & 4.83 \\
\hline$S_{4}-$ Bentonite & 5.39 & 5.19 & 5.29 \\
\hline S.Em \pm & 0.18 & 0.18 & 0.13 \\
\hline C.D. at 5\% & 0.53 & 0.53 & 0.37 \\
\hline \multicolumn{4}{|l|}{ Sulphur levels (kg S/ha) } \\
\hline$L_{1}-10$ & 4.98 & 4.78 & 4.88 \\
\hline $\mathbf{L}_{2}-\mathbf{2 0}$ & 5.58 & 5.38 & 5.48 \\
\hline $\mathbf{L}_{3}-\mathbf{3 0}$ & 5.33 & 5.13 & 5.23 \\
\hline S.Em \pm & 0.16 & 0.16 & 0.11 \\
\hline C.D. at $5 \%$ & 0.46 & 0.46 & 0.32 \\
\hline
\end{tabular}

Table.4 Combined effect of sulphur sources and their levels on no. of branches per plant of soybean

\begin{tabular}{|c|c|c|c|}
\hline \multirow[t]{2}{*}{ Treatments } & \multicolumn{3}{|c|}{ No. of branches plant ${ }^{-1}$} \\
\hline & 2018 & 2019 & Pooled \\
\hline$T_{1}:$ Control & 4.47 & 4.40 & 4.43 \\
\hline$T_{2}: S_{1} L_{1}$ & 4.60 & 4.40 & 4.50 \\
\hline$T_{3}: S_{1} L_{2}$ & 5.87 & 5.67 & 5.77 \\
\hline$T_{4}: S_{1} L_{3}$ & 4.83 & 4.63 & 4.73 \\
\hline$T_{5}: S_{2} L_{1}$ & 5.33 & 5.13 & 5.23 \\
\hline$T_{6}: S_{2} L_{2}$ & 6.20 & 6.00 & 6.10 \\
\hline $\mathbf{T}_{7}: \mathbf{S}_{2} \mathbf{L}_{3}$ & 5.80 & 5.60 & 5.70 \\
\hline $\mathbf{T}_{8}: \mathbf{S}_{3} \mathbf{L}_{1}$ & 4.97 & 4.77 & 4.87 \\
\hline $\mathbf{T}_{9}: \mathbf{S}_{3} \mathbf{L}_{2}$ & 4.63 & 4.43 & 4.53 \\
\hline$T_{10}: S_{3} L_{3}$ & 5.20 & 5.00 & 5.10 \\
\hline $\mathbf{T}_{11}: \mathbf{S}_{4} \mathbf{L}_{1}$ & 5.03 & 4.83 & 4.93 \\
\hline$T_{12}: S_{4} L_{2}$ & 5.63 & 5.43 & 5.53 \\
\hline$T_{13}: S_{4} L_{3}$ & 5.50 & 5.30 & 5.40 \\
\hline \multicolumn{4}{|c|}{ S x L Interaction } \\
\hline S.Em \pm & 0.32 & 0.32 & 0.22 \\
\hline C.D. at 5\% & NS & NS & 0.64 \\
\hline \multicolumn{4}{|c|}{ Control v/s Rest } \\
\hline S.Em \pm & 0.23 & 0.23 & 0.16 \\
\hline C.D. at 5\% & 0.67 & 0.68 & 0.46 \\
\hline C.V. \% & 10.30 & 10.70 & 10.50 \\
\hline \multicolumn{4}{|l|}{$\mathbf{Y} \times \mathbf{T}$} \\
\hline S.Em \pm & \multicolumn{3}{|c|}{0.31} \\
\hline C.D. at 5\% & \multicolumn{3}{|c|}{ NS } \\
\hline
\end{tabular}


Table.5 Mean effect of sulphur sources and their levels on no. of pods per plant of soybean

\begin{tabular}{|c|c|c|c|}
\hline \multirow[t]{2}{*}{ Treatments } & \multicolumn{3}{|c|}{ No. of pods plant ${ }^{-1}$} \\
\hline & 2018 & 2019 & Pooled \\
\hline \multicolumn{4}{|l|}{ Sulphur sources } \\
\hline $\mathbf{S}_{1}-\mathbf{G y p s u m}$ & 39.63 & 41.73 & 40.68 \\
\hline$S_{2}-$ Cosavet Fertis & 41.30 & 43.29 & 42.29 \\
\hline$S_{3}-$ Elemental sulphur & 39.07 & 41.06 & 40.06 \\
\hline$S_{4}-$ Bentonite & 40.73 & 42.83 & 41.78 \\
\hline S.Em \pm & 0.57 & 0.56 & 0.40 \\
\hline C.D. at 5\% & 1.66 & 1.65 & 1.14 \\
\hline \multicolumn{4}{|l|}{ Sulphur levels (kg S/ha) } \\
\hline $\mathbf{L}_{1}-10$ & 39.12 & 41.14 & 40.13 \\
\hline $\mathbf{L}_{2}-\mathbf{2 0}$ & 41.08 & 43.09 & 42.08 \\
\hline $\mathbf{L}_{3}-\mathbf{3 0}$ & 40.35 & 42.45 & 41.40 \\
\hline S.Em \pm & 0.49 & 0.49 & 0.35 \\
\hline C.D. at 5\% & 1.44 & 1.43 & 0.99 \\
\hline
\end{tabular}

Table.6 Combined effect of sulphur sources and their levels on no. of pods per plant of soybean

\begin{tabular}{|c|c|c|c|}
\hline \multirow[t]{2}{*}{ Treatments } & \multicolumn{3}{|c|}{ No. of pods plant ${ }^{-1}$} \\
\hline & 2018 & 2019 & Pooled \\
\hline$T_{1}$ : Control & 34.73 & 36.83 & 35.78 \\
\hline$T_{2}: S_{1} L_{1}$ & 37.63 & 39.73 & 38.68 \\
\hline$T_{3}: S_{1} L_{2}$ & 40.87 & 42.97 & 41.92 \\
\hline$T_{4}: S_{1} L_{3}$ & 40.40 & 42.50 & 41.45 \\
\hline $\mathbf{T}_{5}: \mathbf{S}_{2} \mathbf{L}_{1}$ & 40.20 & 41.97 & 41.08 \\
\hline$T_{6}: S_{2} L_{2}$ & 42.13 & 44.23 & 43.18 \\
\hline $\mathbf{T}_{7}: \mathbf{S}_{2} \mathbf{L}_{3}$ & 41.57 & 43.67 & 42.62 \\
\hline$T_{8}: S_{3} L_{1}$ & 39.13 & 41.23 & 40.18 \\
\hline$T_{9}: S_{3} L_{2}$ & 39.90 & 41.67 & 40.78 \\
\hline$T_{10}: S_{3} L_{3}$ & 38.17 & 40.27 & 39.22 \\
\hline$T_{11}: S_{4} L_{1}$ & 39.53 & 41.63 & 40.58 \\
\hline$T_{12}: S_{4} L_{2}$ & 41.40 & 43.50 & 42.45 \\
\hline$T_{13}: S_{4} L_{3}$ & 41.27 & 43.37 & 42.32 \\
\hline \multicolumn{4}{|c|}{ S x L Interaction } \\
\hline S.Em \pm & 0.98 & 0.98 & 0.69 \\
\hline C.D. at 5\% & NS & $\mathrm{NS}$ & NS \\
\hline \multicolumn{4}{|c|}{ Control v/s Rest } \\
\hline S.Em \pm & 0.76 & 0.75 & 0.53 \\
\hline C.D. at 5\% & 2.21 & 2.20 & 1.50 \\
\hline C.V. \% & 4.23 & 4.01 & 4.12 \\
\hline \multicolumn{4}{|l|}{$\mathbf{Y} \times \mathbf{T}$} \\
\hline S.Em \pm & \multicolumn{3}{|c|}{1.01} \\
\hline C.D. at 5\% & \multicolumn{3}{|c|}{ NS } \\
\hline
\end{tabular}


Table.7 Mean effect of sulphur sources and their levels on seed yield $\left(\mathrm{kg} \mathrm{ha}^{-1}\right)$ of soybean

\begin{tabular}{|c|c|c|c|}
\hline \multirow[t]{2}{*}{ Treatments } & \multicolumn{3}{|c|}{ Seed yield (kg ha $\left.{ }^{-1}\right)$} \\
\hline & 2018 & 2019 & Pooled \\
\hline \multicolumn{4}{|l|}{ Sulphur sources } \\
\hline $\mathbf{S}_{1}-$ Gypsum & 2172 & 2205 & 2189 \\
\hline $\mathbf{S}_{2}-$ Cosavet Fertis & 2323 & 2411 & 2367 \\
\hline$S_{3}-$ Elemental sulphur & 2112 & 2173 & 2143 \\
\hline$S_{4}-$ Bentonite & 2200 & 2238 & 2219 \\
\hline S.Em \pm & 49 & 52 & 36 \\
\hline C.D. at 5\% & 145 & 151 & 102 \\
\hline \multicolumn{4}{|l|}{ Sulphur levels (kg S/ha) } \\
\hline $\mathbf{L}_{1}-\mathbf{1 0}$ & 2107 & 2159 & 2133 \\
\hline $\mathbf{L}_{2}-\mathbf{2 0}$ & 2267 & 2320 & 2294 \\
\hline $\mathbf{L}_{3}-\mathbf{3 0}$ & 2232 & 2291 & 2262 \\
\hline S.Em \pm & 43 & 45 & 31 \\
\hline C.D. at $5 \%$ & 125 & 131 & 88 \\
\hline
\end{tabular}

Table.8 Combined effect of sulphur sources and their levels on seed yield $\left(\mathrm{kg} \mathrm{ha}^{-1}\right)$ of soybean

\begin{tabular}{|c|c|c|c|}
\hline \multirow[t]{2}{*}{ Treatments } & \multicolumn{3}{|c|}{ Seed yield $\left(\mathrm{kg} \mathrm{ha}^{-1}\right)$} \\
\hline & 2018 & 2019 & Pooled \\
\hline$T_{1}$ : Control & 1928 & 1936 & 1932 \\
\hline$T_{2}: S_{1} L_{1}$ & 2031 & 2029 & 2030 \\
\hline$T_{3}: S_{1} L_{2}$ & 2363 & 2433 & 2398 \\
\hline$T_{4}: S_{1} L_{3}$ & 2124 & 2154 & 2139 \\
\hline $\mathbf{T}_{5}: \mathbf{S}_{2} \mathbf{L}_{1}$ & 2208 & 2356 & 2282 \\
\hline$T_{6}: S_{2} L_{2}$ & 2383 & 2411 & 2397 \\
\hline $\mathbf{T}_{7}: \mathbf{S}_{2} \mathbf{L}_{3}$ & 2377 & 2466 & 2421 \\
\hline$T_{8}: S_{3} L_{1}$ & 2154 & 2233 & 2194 \\
\hline$T_{9}: S_{3} L_{2}$ & 1980 & 1982 & 1981 \\
\hline$T_{10}: S_{3} L_{3}$ & 2204 & 2304 & 2254 \\
\hline $\mathbf{T}_{11}: \mathbf{S}_{4} \mathbf{L}_{1}$ & 2033 & 2017 & 2025 \\
\hline$T_{12}: S_{4} L_{2}$ & 2343 & 2456 & 2400 \\
\hline$T_{13}: S_{4} L_{3}$ & 2224 & 2241 & 2232 \\
\hline \multicolumn{4}{|c|}{ S x L Interaction } \\
\hline S.Em \pm & 86 & 89 & 62 \\
\hline C.D. at 5\% & NS & 262 & 176 \\
\hline \multicolumn{4}{|c|}{ Control v/s Rest } \\
\hline S.Em \pm & 61 & 66 & 44 \\
\hline C.D. at 5\% & 178 & 192 & 126 \\
\hline C.V. \% & 9.90 & 9.02 & 9.73 \\
\hline \multicolumn{4}{|l|}{$\mathbf{Y} \times \mathbf{T}$} \\
\hline S.Em \pm & \multicolumn{3}{|c|}{85} \\
\hline C.D. at 5\% & \multicolumn{3}{|c|}{ NS } \\
\hline
\end{tabular}


Table.9 Mean effect of sulphur sources and their levels on straw yield $\left(\mathrm{kg} \mathrm{ha}^{-1}\right)$ of soybean

\begin{tabular}{|c|c|c|c|}
\hline Treatments & 2018 & 2019 & Pooled \\
\hline \multicolumn{4}{|l|}{ Sulphur sources } \\
\hline $\mathbf{S}_{1}-\mathbf{G y p s u m}$ & 2579 & 2711 & 2645 \\
\hline $\mathbf{S}_{2}-$ Cosavet Fertis & 2791 & 2934 & 2862 \\
\hline $\mathbf{S}_{3}-$ Elemental sulphur & 2533 & 2722 & 2627 \\
\hline$S_{4}-$ Bentonite & 2482 & 2623 & 2553 \\
\hline S.Em \pm & 53 & 48 & 36 \\
\hline C.D. at $5 \%$ & 154 & 140 & 101 \\
\hline \multicolumn{4}{|l|}{ Sulphur levels (kg S/ha) } \\
\hline $\mathbf{L}_{1}-10$ & 2485 & 2660 & 2572 \\
\hline $\mathbf{L}_{2}-\mathbf{2 0}$ & 2693 & 2770 & 2731 \\
\hline $\mathbf{L}_{3}-\mathbf{3 0}$ & 2611 & 2813 & 2712 \\
\hline S.Em \pm & 46 & 41 & 31 \\
\hline C.D. at $5 \%$ & 134 & 121 & 88 \\
\hline
\end{tabular}

Table.10 Combined effect of sulphur sources and their levels on straw yield $\left(\mathrm{kg} \mathrm{ha}^{-1}\right)$ of soybean

\begin{tabular}{|c|c|c|c|}
\hline Treatments & 2018 & 2019 & Pooled \\
\hline $\mathbf{T}_{1}$ Control & 2190 & 2340 & 2265 \\
\hline $\mathbf{T}_{2} \mathbf{S}_{1} \mathbf{L}_{1}$ & 2470 & 2509 & 2489 \\
\hline $\mathbf{T}_{3} \mathbf{S}_{1} \mathbf{L}_{2}$ & 2665 & 2910 & 2788 \\
\hline $\mathbf{T}_{4} \mathbf{S}_{1} \mathbf{L}_{3}$ & 2601 & 2714 & 2658 \\
\hline $\mathbf{T}_{5} \mathbf{S}_{2} \mathbf{L}_{1}$ & 2574 & 2825 & 2700 \\
\hline $\mathbf{T}_{6} \mathbf{S}_{2} \mathbf{L}_{2}$ & 3040 & 2828 & 2934 \\
\hline $\mathbf{T}_{7} \mathbf{S}_{2} \mathbf{L}_{3}$ & 2760 & 3147 & 2953 \\
\hline $\mathbf{T}_{8} \mathbf{S}_{3} \mathbf{L}_{1}$ & 2348 & 2620 & 2484 \\
\hline $\mathbf{T}_{9} \mathbf{S}_{3} \mathbf{L}_{2}$ & 2725 & 2878 & 2802 \\
\hline $\mathbf{T}_{10} \mathbf{S}_{\mathbf{3}} \mathbf{L}_{3}$ & 2525 & 2667 & 2596 \\
\hline $\mathbf{T}_{11} \mathbf{S}_{4} \mathbf{L}_{1}$ & 2546 & 2684 & 2615 \\
\hline $\mathbf{T}_{12} \mathbf{S}_{4} \mathbf{L}_{2}$ & 2341 & 2462 & 2401 \\
\hline $\mathbf{T}_{13} \mathbf{S}_{4} \mathbf{L}_{3}$ & 2559 & 2724 & 2641 \\
\hline \multicolumn{4}{|c|}{ S x L Interaction } \\
\hline S.Em \pm & 91 & 83 & 62 \\
\hline C.D. at $5 \%$ & 267 & 243 & 175 \\
\hline \multicolumn{4}{|c|}{ Control v/s Rest } \\
\hline S.Em \pm & 66 & 61 & 44 \\
\hline C.D. at $5 \%$ & 194 & 177 & 126 \\
\hline C.V. \% & 9.12 & 9.25 & 8.97 \\
\hline \multicolumn{4}{|l|}{$\mathbf{Y} \times \mathbf{T}$} \\
\hline S.Em \pm & \multicolumn{3}{|c|}{87} \\
\hline C.D. at $5 \%$ & \multicolumn{3}{|c|}{ NS } \\
\hline
\end{tabular}


Fig.1 General view of the soybean experimental plot

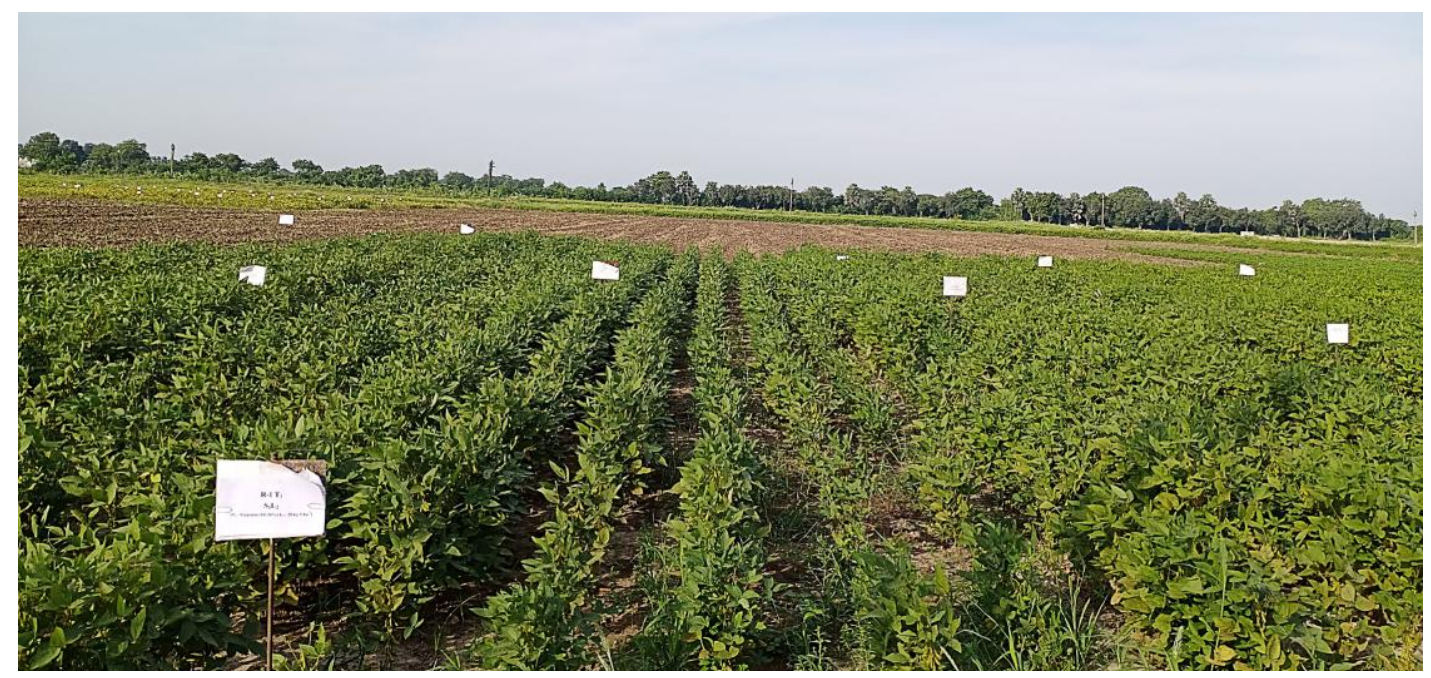

The no. of pods per plant was recorded maximum (42.13, 44.23 and 43.18) under application of cosavet fertis S @ $20 \mathrm{~kg} \mathrm{ha}^{-1}$ $\left(\mathrm{S}_{2} \mathrm{~L}_{2}\right)$. It was statistically at par with application of gypsum at $\mathrm{S} @ 20$ and $30 \mathrm{~kg} \mathrm{~S}$ ha $^{-1}\left(S_{1} L_{2}\right.$ and $\left.S_{1} L_{3}\right)$, cosavet fertis@ 10 and $30 \mathrm{~kg} \mathrm{~S} \mathrm{ha}{ }^{-1}\left(\mathrm{~S}_{2} \mathrm{~L}_{1}\right.$ and $\left.\mathrm{S}_{2} \mathrm{~L}_{3}\right)$ and bentonite @ 20 and $30 \mathrm{~kg} \mathrm{~S} \mathrm{ha}{ }^{-1}\left(\mathrm{~S}_{4} \mathrm{~L}_{2}\right.$ and $\left.\mathrm{S}_{4} \mathrm{~L}_{3}\right)$ in year 2018. While, in year $2019, \mathrm{~S}_{2} \mathrm{~L}_{2}$ was reaminig at par with application of gypsum at $\mathrm{S} @ 20$ and $30 \mathrm{~kg} \mathrm{ha}^{-1}\left(\mathrm{~S}_{1} \mathrm{~L}_{2}\right.$ and $\left.\mathrm{S}_{1} \mathrm{~L}_{3}\right)$, cosavet fertis at $\mathrm{S} @ 30 \mathrm{~kg} \mathrm{ha}^{-1}\left(\mathrm{~S}_{2} \mathrm{~L}_{3}\right)$ and bentonite at $\mathrm{S} @$ 20 and $30 \mathrm{~kg} \mathrm{ha}^{-1}\left(\mathrm{~S}_{4} \mathrm{~L}_{2}\right.$ and $\left.\mathrm{S}_{4} \mathrm{~L}_{3}\right)$. The application of gypsum $\mathrm{S} @ 20 \mathrm{~kg} \mathrm{ha}^{-1}\left(\mathrm{~S}_{1} \mathrm{~L}_{2}\right)$, cosavet fertis $\mathrm{S} @ 30 \mathrm{~kg} \mathrm{ha}^{-1}\left(\mathrm{~S}_{2} \mathrm{~L}_{3}\right)$ and bentonite $\mathrm{S} @ 20$ and $30 \mathrm{~kg} \mathrm{ha}^{-1}\left(\mathrm{~S}_{4} \mathrm{~L}_{2}\right.$ and $\mathrm{S}_{4} \mathrm{~L}_{3}$ ) were found at par in pooled result (Table 6).

\section{Seed yield}

The application of cosavet fertis $\left(S_{2}\right)$ produced significantly highest seed yield (2323, 2411 and $2367 \mathrm{~kg} \mathrm{ha}^{-1}$ ) as compare to other sources during year 2018, 2019 and pooled result, respectively (Table 7 ). It was statistically at par $\left(2200 \mathrm{~kg} \mathrm{ha}^{-1}\right)$ with application of bentonite $\left(\mathrm{S}_{4}\right)$ during year 2018. The results confirmed to reports of Singh et al., (2018). While, The significantly the highest seed yield was registered at $20 \mathrm{~kg}$ $\mathrm{S} \mathrm{ha}^{-1}\left(\mathrm{~L}_{2}\right)(2267,2320$ and 2294) during both years and pooled result, respectively. It was found at par (2232, 2291 and $2262 \mathrm{~kg} \mathrm{ha}^{-1}$ ) with sulphur at $30 \mathrm{~kg} \mathrm{~S} \mathrm{ha}^{-1}\left(\mathrm{~L}_{3}\right)$. The present findings are in close agreement with the results obtained by Mamatha et al., (2018).

The interaction effect of sulphur sources and their levels on seed yield was found significant (Table 8). The maximum seed yield (2466 and $2421 \mathrm{~kg} \mathrm{ha}^{-1}$ ) was observed under application of cosavet fertis @ $30 \mathrm{~kg} \mathrm{~S}$ $\mathrm{ha}^{-1}\left(\mathrm{~S}_{2} \mathrm{~L}_{3}\right)$ recorded during year 2019 and pooled result, respectively. It was statistically (2466 kg ha ${ }^{-1}$ ) at par under application of gypsum @ $20 \mathrm{~kg} \mathrm{~S} \mathrm{ha}^{-1}\left(\mathrm{~S}_{1} \mathrm{~L}_{2}\right)$, cosavet fertis @ 10 and $20 \mathrm{~kg} \mathrm{~S}^{-1}\left(\mathrm{~S}_{2} \mathrm{~L}_{1}\right.$ and $\left.\mathrm{S}_{2} \mathrm{~L}_{2}\right)$, elemental sulphur@10 and 30 kg S ha ${ }^{-1}$ $\left(\mathrm{S}_{3} \mathrm{~L}_{1}\right.$ and $\left.\mathrm{S}_{3} \mathrm{~L}_{3}\right)$ and bentonite @ 20 and $30 \mathrm{~kg}$ $\mathrm{S}$ ha $^{-1}\left(\mathrm{~S}_{4} \mathrm{~L}_{2}\right.$ and $\left.\mathrm{S}_{4} \mathrm{~L}_{3}\right)$ during year 2019. While, application of gypsum with $20 \mathrm{~kg} \mathrm{~S}$ $\mathrm{ha}^{-1}\left(\mathrm{~S}_{1} \mathrm{~L}_{2}\right)$, cosavet fertis with 10 and $20 \mathrm{~kg} \mathrm{~S}$ ha $^{-1}\left(\mathrm{~S}_{2} \mathrm{~L}_{1}\right.$ and $\left.\mathrm{S}_{2} \mathrm{~L}_{2}\right)$, elemental sulphur with $30 \mathrm{~kg} \mathrm{~S}^{-1}\left(\mathrm{~S}_{3} \mathrm{~L}_{3}\right)$ and bentonite with $20 \mathrm{~kg} \mathrm{~S}$ $\mathrm{ha}^{-1}\left(\mathrm{~S}_{4} \mathrm{~L}_{2}\right)$ were found at par in pooled result. Similar, results were also obtained by Yatheesh et al., (2013).

The combined application of sulphur sources 
and levels (Table 8) significantly influenced on seed yield of soybean as compare to control. The seed yield of soybean was significantly higher $\left(2383 \mathrm{~kg} \mathrm{ha}^{-1}\right)$ with application of sulphur @ $20 \mathrm{~kg} \mathrm{ha}^{-1}$ as a source of cosavet fertis at $\left(\mathrm{S}_{2} \mathrm{~L}_{2}\right)$ during year 2018 and application of cosavet fertis @ 30 $\mathrm{kg} \mathrm{S} \mathrm{ha}{ }^{-1}\left(\mathrm{~S}_{2} \mathrm{~L}_{3}\right)$ produced the highest value of seed yield (2466 and $2421 \mathrm{~kg} \mathrm{ha}^{-1}$ ) during year 2019 and pooled result as compare to control (1928, 1936 and $1932 \mathrm{~kg} \mathrm{ha}^{-1}$ ), respectively. It was remaining at par with application of gypsum @ $20 \mathrm{~kg} \mathrm{~S} \mathrm{ha}^{-1}\left(\mathrm{~S}_{1} \mathrm{~L}_{2}\right)$, cosavet fertis@10 and $30 \mathrm{~kg} \mathrm{Sha}^{-1}\left(\mathrm{~S}_{2} \mathrm{~L}_{1}\right.$ and $\left.\mathrm{S}_{2} \mathrm{~L}_{3}\right)$ and bentonite @ 20 and $30 \mathrm{~kg} \mathrm{~S} \mathrm{ha}^{-1}$ $\left(\mathrm{S}_{4} \mathrm{~L}_{2}\right.$ and $\left.\mathrm{S}_{4} \mathrm{~L}_{3}\right)$ during year 2018. The application of gypsum @ $20 \mathrm{~kg} \mathrm{~S} \mathrm{ha}^{-1}\left(\mathrm{~S}_{1} \mathrm{~L}_{2}\right)$, cosavet fertis@10 and $20 \mathrm{~kg} \mathrm{~S} \mathrm{ha}^{-1}\left(\mathrm{~S}_{2} \mathrm{~L}_{1}\right.$ and $\mathrm{S}_{2} \mathrm{~L}_{2}$ ), elemental sulphur @ $30 \mathrm{~kg} \mathrm{~S} \mathrm{ha}{ }^{-1}$ $\left(\mathrm{S}_{3} \mathrm{~L}_{3}\right)$ and bentonite @ $20 \mathrm{~kg} \mathrm{~S} \mathrm{ha}{ }^{-1}\left(\mathrm{~S}_{4} \mathrm{~L}_{2}\right)$ was found at par with application of cosavet fertis at $30 \mathrm{~kg} \mathrm{Sha}^{-1}\left(\mathrm{~S}_{2} \mathrm{~L}_{3}\right)$ were found during year 2019 and application of gypsum @ $20 \mathrm{~kg}$ $\mathrm{S} \mathrm{ha}^{-1}\left(\mathrm{~S}_{1} \mathrm{~L}_{2}\right)$, cosavet fertis@ $20 \mathrm{~kg} \mathrm{~S} \mathrm{ha}^{-1}$ $\left(\mathrm{S}_{2} \mathrm{~L}_{2}\right)$ and bentonite @ $20 \mathrm{~kg} \mathrm{~S} \mathrm{ha}{ }^{-1}\left(\mathrm{~S}_{4} \mathrm{~L}_{2}\right)$ were remaining at par in pooled result.

\section{Straw yield}

A significant increased in straw yield was observed under different sources of sulphur application (Table 9). The significantly the highest straw yield (2791, 2934 and $2862 \mathrm{~kg}$ $\mathrm{ha}^{-1}$ ) was registered at application of cosavet fertis $\left(S_{2}\right)$ as compared to other sources of sulphur in both years and pooled, respectively. Similar results were reported by Yadav et al., 2018. While, the application of sulphur at $20 \mathrm{~kg} \mathrm{~S} \mathrm{ha}^{-1}\left(\mathrm{~L}_{2}\right)$ produced the highest straw yield (2693 and $2731 \mathrm{~kg} \mathrm{ha}^{-1}$ ) during year 2018 and pooled result, respectively. It was statistically at par (2611 and $2712 \mathrm{~kg} \mathrm{ha}^{-1}$ ) with application of sulphur at $30 \mathrm{~kg} \mathrm{ha}^{-1}\left(\mathrm{~L}_{3}\right)$. While, the application of sulphur at $30 \mathrm{~kg} \mathrm{ha}^{-1}\left(\mathrm{~L}_{3}\right)$ noted highest straw yield $\left(2813 \mathrm{~kg} \mathrm{ha}^{-1}\right)$ and was at par $(2770 \mathrm{~kg}$ $\mathrm{ha}^{-1}$ ) with application of sulphur at $20 \mathrm{~kg} \mathrm{ha}^{-1}$ $\left(\mathrm{L}_{2}\right)$ during year 2019. The results were in close agreement with Hosmath et al., (2014).

The interaction effect of application of sulphur sources and their levels on straw yield was found significant (Table 10). The application of cosavet fertis @ $20 \mathrm{~kg} \mathrm{ha}^{-1}$ $\left(\mathrm{S}_{2} \mathrm{~L}_{2}\right)$ recorded maximum straw yield (3040 $\mathrm{kg} \mathrm{ha}^{-1}$ ) during year 2018 and application of cosavet fertis@30 kg ha ${ }^{-1}\left(\mathrm{~S}_{2} \mathrm{~L}_{3}\right)$ produced the highest straw yield (3147 and 2953 $\mathrm{kg} \mathrm{ha}^{-1}$ ) during year 2019 and pooled result, respectively. It was remain at par with application of gypsum with $20 \mathrm{~kg} \mathrm{~S}$ ha $^{-1}$ $\left(\mathrm{S}_{1} \mathrm{~L}_{2}\right)$ during year 2019 . The application of gypsum with $20 \mathrm{~kg} \mathrm{~S}$ ha $^{-1}\left(\mathrm{~S}_{1} \mathrm{~L}_{2}\right)$, cosavet fertis with $20 \mathrm{~kg} \mathrm{~S} \mathrm{ha}^{-1}\left(\mathrm{~S}_{2} \mathrm{~L}_{2}\right)$ and elemental sulphur with $20 \mathrm{~kg} \mathrm{~S}^{-1}\left(\mathrm{~S}_{3} \mathrm{~L}_{2}\right)$ were remain at par in pooled result. Similar result was also noted by Verma et al., (2013).

The combined application of sulphur sources and levels (Table 10) significantly influenced on straw yield of soybean as compare to control. The significantly highest straw yield (3040 kg ha ${ }^{-1}$ ) was registered with application of cosavet fertis@20 kg S ha ${ }^{-1}\left(\mathrm{~S}_{2} \mathrm{~L}_{2}\right)$ during year 2018 and application of cosavet fertis @ $30 \mathrm{~kg} \mathrm{~S} h a^{-1}\left(\mathrm{~S}_{2} \mathrm{~L}_{3}\right)$ produced the highest straw yield (3147 and $2953 \mathrm{~kg} \mathrm{ha}^{-1}$ ) during year 2019 and pooled result over that of control (2190, 2340 and $\left.2265 \mathrm{~kg} \mathrm{ha}^{-1}\right)$, respectively. It was found at par with application of cosavet fertis@20 kg S ha ${ }^{-1}\left(\mathrm{~S}_{2} \mathrm{~L}_{2}\right)$ in pooled result.

The favorable effect of sulphur fertilization on yield components and finally on yield might be due to balanced nutritional environment, efficient and greater partitioning of metabolites and adequate translocation of nutrients towards reproductive site.

The increase in seed yield may be due to stimulatory effect of applied sulphur on the synthesis of protein, which in turn might have 
accelerated photosynthesis and improved most of the yield contributing characters which resulted in significantly higher seed yield (Tulasi et al., 2014). Increased in straw yield due to increased growth, which resulted in increased photosynthesis and assimilation rates, cell division, cell elongation and activation of enzymes which in turn increased straw yield (Poomurugesan and Poonkodi, 2008). Judicial supply of sulphur along with sources, contributes to better growth, thereby affectively increasing the yield per hectare.

Based on the results, it can be concluded that the application of sulphur in form of cosavet fertis at $20 \mathrm{~kg} \mathrm{ha}^{-1}$ significantly improved the yield and yield attributes of soybean. It is found efficient for higher and qualitative yield production of soybean.

\section{Acknowledgement}

The authors are heartly thankful to Department of Agricultural Chemistry and Soil Science, College of Agriculture, Junagadh Agricultural University, Junagadh, India for providing instrumental facilities and necessary facilities during the course of investigation.

\section{References}

Gupta V, Sharma GL, Sonakiya VK, Tiwari G (2003). Impact of different levels of FYM and sulphur on morphophysiological indices and productivity of soybean genotypes. Jawaharlal Nehru Krishi Vishwa Vidyalaya, Jabalpur. 37(2):76-78.

Hosmath JA, Babalad HB, Basavaraj GT, Jahagirdar S, Patil RH, Athoni BK, Agasimani SC (2014). Sulphur nutrition in soybean [Glycine max (L.) Merril] in India. International Conference on Biological, Civil and Environmental Engineering (BCEE-2014) March 2017-
18, Dubai (UAE).

Kumar V, Singh M, Singh N (1981). Effect of sulphur, phosphorus and molybdate application on quality of soybean grain. Plant and Soil. 59(1):3-8.

Layek J, Shivakumar BG, Rana DS, Gangaiah B, Lakshman K, Paramanik B (2014). Growth, nodulation, physiological indices and yield of soybean as influenced by sulphur and boron nutition. An International Journal of Life Science. 9(4):1389-1393.

Mamatha N, Chandra Shaker K, Padmaja G, Reddy MM (2018). Influence of nitrogen and sulphur application on yield and quality of soybean (Glycine $\max$ L.). International Journal of Current Microbiology and Applied Sciences. 7(12): 3452-3457.

Panse VG and Sukhatme PV ( 1985). Statistical Methods for Agricultural Workers. Indian Council of Agricultural Research, New Delhi.

Poomurugesan AV and Poonkodi P (2008). Effect of sources and levels of sulphur on growth and yield performances of sunflower (Helianthus annus). Mysore Journal of Agricultural Science. 42(1):147-153.

Singh G, Pathania P, Rana SS, Kumar S, Sharma VK (2018). Response of soybean to levels and sources of sulphur on growth and yield under mid - hill conditions of Himachal. International Journal of Chemical Studies. 6(6):29032907.

Singh SP, Kumar Y, Singh S (2017). Effect of sources and levels of sulphur on yield, quality and uptake of nutrients in green gram (Vigna radiata). Annals of Plant and Soil Research. 19(2):143-147.

Tandon HLS ( 1987). Sulphur research and Agricultural production in India, FDCO, New Delhi ( $2^{\text {nd }}$ edition).

Tulasi LT, Nawlakhe SM, Mankar DD, Shrinivasrao M, Gauri VB (2014). 
Growth, yield and quality of summer sesame as influenced by the fertilizer and sulphur levels. Journal of Soils and Crops. 24(1):143-147.

Vaiyapuri K, Mohamed AM, Rajendran K (2010). Influence of sulphur and boron on yield attributes and yield of soybean. Madras Agricultural Journal. 97(13):65-67.

Verma SK, Raghuwanshi SRS, Tiwari SC, Jain R (2013). Effect of gypsum as a source of sulphur on soybean [Glycine $\max ($ L.) Merrill] at farmer's field on Malwa region of Madhya Pradesh. Soybean Research. 11(1): 29-35.

Vyas MD and Khandwe R (2013). Effect of sulphur and boron levels on productivity, quality and profitability of soybean [Glycine max (L.) Merrill] in Vertisols under rainfed conditons. Soybean Research. 11(1): 14-21.

Yadav SK, Mahto DK, Chaudhary SK (2018). Influenced of different levels and sources of sulphur on nodulation and quality of blackgram [Vigna mungo (L.) Hepper]. Journal of Pharmacognosy and Phytochemistry, SP1: 2027-2029.

Yatheesh G, Gurumurthy KT, Sridhara CJ, Prakasha HC (2013). Effect of different sources and levels of sulphur on growth and yield of soybean (Glycine max L.). Mysore Journal of Agricultural Sciences. 47(3): 592-595.

\section{How to cite this article:}

Janaki Movalia and Savalia, S. G. 2020. Effect of Sources and Levels of Sulphur on Growth and Yield of kharif Soybean [Glycine $\max$ (L.) Merrill]. Int.J.Curr.Microbiol.App.Sci. 9(10): 440-451. doi: https://doi.org/10.20546/ijcmas.2020.910.054 